\title{
Self - perceived and clinically diagnosed dental and periodontal health status among young adults and their implications for epidemiological surveys Yuval Vered* and Harold D Sgan-Cohen
}

Address: Department of Community Dentistry, Faculty of Dental Medicine, Hebrew University-Hadassah School of Dental Medicine, Jerusalem, Israel

Email: Yuval Vered* - vyuval@hadassah.org.il; Harold D Sgan-Cohen - harolds@cc.huji.ac.il

* Corresponding author

Published: 13 July 2003

BMC Oral Health 2003, 3:3
Received: 08 April 2003

Accepted: I 3 July 2003

This article is available from: http://www.biomedcentral.com/I472-683I/3/3

(c) 2003 Vered and Sgan-Cohen; licensee BioMed Central Ltd. This is an Open Access article: verbatim copying and redistribution of this article are permitted in all media for any purpose, provided this notice is preserved along with the article's original URL.

\begin{abstract}
Background: Clinical (normative) and subjective (self-assessment) evaluation of caries and periodontal diseases have been reported to demonstrate a significant disparity. The dental public health team is obligated to recognize and understand this gap. The objectives of the study were to investigate the practical values of using questionnaires (self-perceived assessment) as compared to clinical examinations (normative assessment) and to evaluate the implications of the results in understanding the public's perception of oral health.
\end{abstract}

Methods: The investigation was performed on 4920, 21 year-old Israeli adults upon release from compulsory military service between 1996 and 1998. Participants were asked to fill in a questionnaire inquiring how they would rate their personal dental and periodontal health levels. Clinical examinations, employing the DMFT and CPITN indices, were performed to determine normative oral health status. Perceived and normative assessments were compared for sensitivity, specificity, positive and negative predictive values and overall proportions using the clinical examinations as a gold standard.

Results: The sensitivity (disease perception) for dental status was found to be 0.34 , while the specificity (health perception) was found to be 0.83 . The positive predictive value for perceived dental status was found to be 0.68 , whereas the negative predictive value was found to be 0.54 . The sensitivity for perceived periodontal status was found to be 0.28 , while the specificity was found to be 0.83 . The positive predictive value for perceived periodontal status was found to be 0.05 , whereas the negative predictive value was found to be 0.97 . Regarding the overall proportions, a large discrepancy was found between self-assessment and professional assessment for both dental and periodontal health status.

Conclusions: Self-assessment questionnaires were of low value in evaluating oral health status both in the individual and public levels, though perception levels of health were higher than that of disease. Findings reflects a low level of awareness of the public that may influence care-seeking behavior and highlight the importance of oral health promotion and the crucial need for public health action. 


\section{Background}

Caries and periodontal diseases, the two foremost oral pathologies, remain widely prevalent and affect all populations throughout the life span [1]. Clinical (normative) assessment of these pathologies is an expensive, invasive and uncomfortable procedure for many people. Recently, medicolegal and ethical obstacles have further complicated these procedures [2,3]. Consequently, the use of questionnaires and interviews (self-perceived assessment) have become a more common method for collecting diagnostic data and performing oral health surveys [2-4]. Comparisons of oral health normative versus selfperceived assessments could demonstrate the efficacy of the individual to evaluate personal health status and highlight fields in which self-perceived assessment is precise or unprecise [2]. Studies comparing questionnaires and interviews with clinical examinations have demonstrated the efficacy of self-perceived assessment concerning the number of teeth and presence of dentures [4-6]. Self-perceived assessment data have neither been found as useful nor successful in the assessment of individual dental and periodontal health status [2-4,7]. Kallio et al. [7] suggested that self-reporting of gingival health lacks sufficient validity in the screening of individuals for gingivitis. Heloe [4] found that gingival disease was markedly underreported by self-assessment. Gilbert et al. [3] compared questionnaires and clinical assessment of periodontal health status, and the results yielded sensitivity and specificity values of below 50\%. Trevonen et al. [8] reported that people tend to underestimate their dental treatment needs, mainly in the field of periodontology. In regard to the presence of dental caries, Robinson et al. [2], compared questionnaire and clinical assessment, and the results yielded sensitivity and positive predictive values of $58 \%$, and specificity and negative predictive values of $71 \%$. Nevertheless, they suggested that the use of questionnaires should be further investigated with reference to community rather than individual aspects.

In Israel, since 1993, oral health assessment has been included in an ongoing national health survey, approved by a human research ethical committee, seeking information on a wide number of health issues. The survey is conducted among a representative sample of 21-year-old soldiers (young adults), upon release from compulsory military service [9]. This population is considered representative in terms of socio-economic, geographic and cultural variables. In addition to the clinical (medical and dental) examinations each participant is asked to answer a self-administered questionnaire, which includes personal, medical and dental issues, as well as self-perceived assessment of dental and periodontal health status. Although participation is non-obligatory, annual response rates are over $90 \%$.
The objectives of the present study were:

- To investigate self-perceived versus clinically diagnosed dental and periodontal health status.

- To assess the value of using questionnaires in large-scale population surveys.

- To evaluate the implications of the results in understanding the public perception of oral health.

\section{Methods}

\section{Study group}

A systematic representative sample included 4920, 21 year-old (Israeli Defense Forces) soldiers upon release from compulsory military service. Sample design was broadly described in a previous study by Sgan-Cohen et al. [9]. Information for the present study was gathered over a three-year period: 1996 to 1998.

\section{Clinical examination}

Each participating soldier was summoned to the dental examination room. Examinations, which were performed in accordance with the WHO standardized methodology [10], were conducted over the study period by five examiners, supervised and calibrated by the same epidemiologist [9]. Dental radiographs were excluded.

\section{Clinical variables}

\section{Dental status}

The DMFT index was employed, using flat dental mirrors and sharp sickle-shaped explorers.

\section{Periodontal status}

The CPITN index was employed, using the WHO periodontal probe.

\section{Questionnaire}

The questionnaire was applied prior to the dental examination, in order to avoid any potential effect of the dentist's diagnosis on the subject's self-perception. Each participant was asked to answer a self-administered questionnaire regarding personal, medical and dental issues. Regarding dental and periodontal health status the following two questions were included for analysis in the present study:

\section{1. "What is your opinion regarding the health status of your teeth?"}

\section{2. "What is your opinion regarding the health status of your gums?"}


Table I: Sensitivity and Specificity for perceived dental status according to different cut-off points.

\begin{tabular}{lll}
\hline Cut-off point & Sensitivity (\%) & Specificity (\%) \\
\hline DMFT = 8 & $34 \%$ & $83 \%$ \\
DMFT = I & $27 \%$ & $90 \%$ \\
D = I & $33 \%$ & $86 \%$ \\
\hline
\end{tabular}

Table 2: Perceived and normative assessment of dental health.

\begin{tabular}{llll}
\hline Normative Perceived & DMFT >8 & DMFT 0-8 & Total \\
\hline Bad & TP 868 & FP 409 & 1277 \\
Good & FN 1664 & TN 1979 & 3643 \\
Total & 2532 & 2388 & 4920 \\
\hline
\end{tabular}

- TP $=$ True Positive $\cdot \mathrm{FP}=$ False Positive $\bullet \mathrm{FN}=$ False Negative $\bullet \mathrm{TN}=$ True Negative $\bullet$ Sensitivity $(\mathrm{TP} /(\mathrm{TP}+\mathrm{FN}))=0.34(95 \% \mathrm{Cl} 0.32-0.36) \cdot \cdot \mathrm{Spe}-$ cificity $(\mathrm{TN} /(\mathrm{TN}+\mathrm{FP}))=0.83(95 \% \mathrm{Cl} 0.8 \mathrm{I}-0 / 83) \cdot$ Positive Predictive Value $(\mathrm{TP} /(\mathrm{TP}+\mathrm{FP}))=0.68 \cdot$ Negative Predictive Value $(\mathrm{TN} /(\mathrm{TN}+\mathrm{FN}))=0.54$ - Overall Proportions = 1277 participants $(26 \%)$ reported having bad dental health status compared to $2532(5 \mid .5 \%)$ clinically caries diagnosed subjects (McNemar test, $p=<0.00000 \mathrm{I}$ ).

Possible answers were "very good", "good", "not so good", and "bad". For research purposes the answers "very good" and "good" were combined and considered to indicate a "Good" self-perceived assessment and the answers "not so good" and "bad" indicated a "Bad" self-perceived assessment for both teeth and gums.

The "gold standard" clinical examinations and the selfperceived assessment questionnaire were compared for:

- Sensitivity - The proportion of individuals who perceive having the disease among those who are clinically diagnosed as having the disease.

- Specificity - The proportion of individuals who perceive not having the disease among those who are clinically diagnosed as not having the disease.

- Positive predictive value (PPV)- The proportion of individuals who have the disease (according to the clinical examination) among the total group that perceive having the disease.

- Negative predictive value (NPV)- The proportion of individuals who do not have the disease (according to the clinical examination) among the total group that perceive not having the disease.

- Overall proportions - The proportions of clinically diagnosed disease among the total population and self-perceived disease among the total population.
Sensitivity and specificity rates are presented with their appropriate 95\% CI (Confidence Intervals). For the relationships between lay and professional assessments, the McNemar test for paired observations was applied. Statistically significant levels were chosen at $\mathrm{p}<0.05$.

\section{Results \\ Dental status}

Four thousand, nine hundred and twenty participants responded to the question about the health status of their teeth.

Three cut-off points were chosen for operational definitions:

1. DMFT $=8$ (in accordance with mean DMFT of $8.34 \pm$ 4.85 of present study participants): DMFT scores $0-8=$ "Good" dental status and DMFT > $8=$ "Bad" dental status,

2. DMFT $=1$ : DMFT score $0=$ "Good" dental status and DMFT $\geq 1$ = "Bad" dental status.

3. $\mathrm{D}$ (component of DMFT) $=1$ : D score $0=$ "Good" dental status and $\mathrm{D} \geq 1$ = "Bad" dental status.

Data regarding the sensitivity and specificity values for different cut-off points are summarized in Table 1.

For DMFT $=8$ cut-off point, Sensitivity was 0.34 and Specificity was 0.83 . For DMFT $=1$ cut-off point, Sensitivity was 0.27 and Specificity was 0.90 . For $\mathrm{D}=1$ cut-off point, Sensitivity was 0.33 and Specificity was 0.86 . 
Table 3: Perceived and normative assessment of periodontal health.

\begin{tabular}{llll}
\hline Normative Perceived & CPITN 3-4 & CPITN 0-2 & Total \\
\hline Bad & TP 46 & FP 77I & 817 \\
Good & FN II8 & TN 3520 & 3638 \\
Total & I64 & 4291 & 4455 \\
\hline
\end{tabular}

- TP $=$ True Positive $\bullet \mathrm{FP}=$ False Positive $\bullet \mathrm{FN}=$ False Negative $\bullet \mathrm{TN}=$ True Negative $\bullet$ Sensitivity $(\mathrm{TP} /(\mathrm{TP}+\mathrm{FN}))=0.28(95 \% \mathrm{Cl} 0.2 \mathrm{I}-0.35) . \cdot \mathrm{Spe}-$ cificity $(T N /(T N+F P))=0.83(95 \% \mathrm{Cl} 0.8 \mathrm{I}-0 / 83) \cdot$ Positive Predictive Value $(T P /(T P+F P))=0.05 \cdot$ Negative Predictive Value $(T N /(T N+F N))=0.97$ - Overall Proportions $=817$ participants $(18 \%)$ reported having bad periodontal health status compared to 164 (3.7\%) clinically periodontal disease diagnosed subjects (McNemar test, $p=<0.000001$ ).

Following these similar results, the mean DMFT $=8$ cutoff point was chosen for further analysis. Data are summarized in Table 2.

According to the clinical examination, 2532 (51.4\%) participants were found to have DMFT>8 and 2388 (48.5\%) participants were found to have DMFT of 8 or less.

According to the questionnaire, 1277 (25.9\%) participants reported to have bad dental health status and 3643 (74\%) participants reported to have good dental health status.

The sensitivity for perceived dental status was found to be 0.34 (95\% CI $0.32-0.36)$, while the specificity was found to be 0.83 ( $95 \%$ CI $0.81-0.83$ ).

The positive predictive value for perceived dental status was found to be 0.68 , whereas the negative predictive value was found to be 0.54 .

Regarding the overall proportions, 26\% (1277) of the participants perceived having bad dental health status compared to $51.5 \%$ (2532) who were clinically found to have bad dental health status ( $\mathrm{p}<0.000001$, McNemar test).

\section{Periodontal status}

Four thousand, four hundred and fifty five participants responded to the question about the health status of their gums.

Operational definitions were:

1. CPITN scores $0-2$ (healthy, bleeding or calculus as the worst score) $=$ "Good" periodontal status.

2. CPITN scores $3-4$ (shallow and deep periodontal pockets, respectively) $=$ "Bad" periodontal status.

Data are summarized in table 3.
According to the clinical examination, 164 (0.036\%) participants were found to have CPITN scores of 3 or 4 and 4291 (96.3\%) participants were found to have CPITN of 0 to 2 .

According to the questionnaire, 817 (18.33\%) participants reported to have bad periodontal health status and 3638 (81.66\%) participants reported to have good periodontal health status.

The sensitivity for periodontal status was found to be 0.28 (95\% CI 0.21-0.35), while the specificity was found to be 0.83 (95\% CI 0.81-0.83).

The positive predictive value for periodontal status was found to be 0.05 , whereas the negative predictive value was found to be 0.97 .

Regarding the overall proportions, $18 \%$ (817) of the participants perceived having bad periodontal health status compared with $3.7 \%$ ((164) who were clinically diagnosed as having bad periodontal health status ( $\mathrm{p}<$ 0.000001, McNemar test).

\section{Discussion \& Conclusion}

Previous studies have shown that questionnaires and interviews that collect data on oral health status are useful measures specifically for ascertaining the presence of dentures and the number of teeth [4-6]. Self-reporting of gingival and periodontal health status as well as selfassessment of the presence of dental caries have been found to be neither useful nor successful $[2-4,7,8]$. Gilbert et al. [3] reported that only four out of eighteen items were weakly predictive of the periodontal health status. They concluded that the values obtained were not sufficient to enable development of a set of questions that would serve as a satisfactory indicator for periodontal condition in the absence of clinical examinations. Robinson et al. [2] suggested that self-reported interview data are not useful for assessing the presence of dental decay. In their study, 26 out of 45 participants who thought they had decay had one or more decayed teeth, 19 out of 65 
participants, who did not think they had caries, were found to have caries by clinical examination. Joshipura et al. [11] suggested that valid self-reported measures could provide a time-and-cost-efficient alternative for large epidemiological studies. As a result, oral health could be included in more studies on overall health status. Pitiphat et al. [12] assessed the validity of self-reported oral disease measures in two populations. In their study, self-reports provided reasonably valid estimates for number of remaining teeth, fillings, root canal therapy, and fixed and removable prostheses. However, they appear to be less useful for the assessment of dental caries and periodontal disease.

In the present study a large and representative population of young adults was investigated. The DMFT and CPITN indices, which were used as the gold standard, are the preferred and recommended indicators as recommended by the WHO for the assessment of dental and periodontal health status in oral health surveys [13].

The clinical examination and self-perceived assessment questionnaires were compared for sensitivity, specificity, positive and negative predictive values and overall proportions. It has been suggested that sensitivity and specificity should be considered together and only when both are high the measure is of adequate validity [14]. Predictive values of positive and negative tests are influenced by the prevalence of the disease in the population, in which the test is performed, so that the lower the prevalence, the lower the predictive value of a positive test will be [14].

Perceived oral health status in the present study was found to be of high specificity ( 0.83 for dental caries status and 0.83 for periodontal status), but low sensitivity ( 0.34 for dental caries status and 0.28 for periodontal status). Additionally, self-perceived oral health status was found to be of moderate positive predictive value and negative predictive value for dental caries status (0.68 and 0.54 respectively) and of very low positive predictive value and very high negative predictive value for periodontal status $(0.05$ and 0.97 respectively. Pitiphat et al. [12], who employed several threshold points for self-report measure of periodontal disease found low sensitivity values of $17.7 \%$ $64.7 \%$, and high specificity values of $59.8 \%-90.7 \%$, similar to the results of the present study.

Regarding the assessment of overall proportions, Robinson et al. [2], have suggested that the use of questionnaires should be investigated in adult populations where individual errors compensate for each other. In their study, the number of participants with caries by self-assessment (responding to the question: " Do you think some of your teeth are decayed"?) was identical to that found with caries by professional assessment and the number of partici- pants in need for treatment by self-assessment was similar to that found by professional assessment. In the present study, in which different questions ("What is your opinion regarding the health status of your teeth and gums"?) were asked, a large discrepancy was found between self-assessment and professional assessment for both dental and periodontal status. Regarding dental health status, $26 \%$ (1277) of the participants reported to be in a "bad" condition compared to clinical examination that identified $51.5 \%$ (2532) to be in a "bad" condition. Regarding periodontal health status, these levels were $18 \%$ (817) and only $3.7 \%$ (164) accordingly.

The present study gives rise to several important issues. It has been noted that people seem to be unable to recognize whether they are affected by dental and periodontal diseases $[2-8,12]$. The comparison performed in the present study suggests that perception of health is at a higher level, whereas perception of disease is much lower. Regarding dental caries status, 1664 participants (45.6\%) out of a total of 3643 who thought they had good dental status, were found to be wrong according to the clinical examination (false negative group). Regarding periodontal health status, the small group of participants with CPITN scores of 3 and 4,164 out of $4455(0.036 \%)$, is due to the young age and the low prevalence of periodontal diseases in this age group. Due to the low positive predictive value, 771 participants $(94.3 \%)$ out of a total of 817 who thought they had bad periodontal status were found to be wrong according to the clinical examination (false positive group).

These findings reflect a high level of unawareness that may influence an oral health care seeking behavior. Utilization of clinical facilities by consumers, although generated by a wide variety of factors, many of them psychosocial, is motivated first and foremost by self-perception of illness [15].

In conclusion, our study showed that questionnaires do have an inherent and significant value in explaining the levels of dental awareness, perception and self-assessment, which in the present study have clearly demonstrated an alarming discrepancy with the "gold standard" of professional clinical assessment.

The key to an informed and motivated public lies in the hands of the profession (private and public health practitioners), as well as the authorities. Health promotion, with its key concepts of equity and equality, empowerment and advocacy, offers a new and often complex approach to improving both general and oral health [16]. It shifts the responsibility for health from the formal health care system to individuals, communities and decision-makers at all levels of society. The professional exam- 
ination and diagnosis should be more cognizant of the public perception, and the profession should be more involved regarding the public dental health felt and expressed needs [17]. In both aspects a crucial need for public health action is clearly evident.

\section{Competing Interests}

None declared.

\section{Authors' Contributions}

Yuval Vered:

1. Collection of data.

2. Statistical analysis.

3. Summary of findings.

4. Presentation and writing of report.

Harold D. Sgan-Cohen:

1. Examiners calibration.

2. Monitoring of clinical exams.

3. Summary of findings.

4. Presentation and writing of report.

\section{References}

I. Barmes DE: A global view of oral diseases: today and tomorrow Community Dent Oral Epidemiol 1999, 27:2-7.

2. Robinson PG, Nadanovsky $P$ and Sheiham A: Can questionnaires replace clinical surveys to assess dental treatment needs of adults? J Public Health Dent 1998, 58:250-3.

3. Gilbert $A D$ and Nuttall NM: Self - reporting of periodontal health status Brit Dent J 1999, I 86:24I-4.

4. Helöe LA: Comparison of dental health data obtained from questionnaires, interviews and clinical examination Scand J Dent Res 1972, 80:495-9.

5. Könönen M, Lipasti J, Murtomaa H: Comparison of dental information obtained from self - examination and clinical examination. Community Dent Oral Epidemiol 1986, I4:258-60.

6. Palmqvist S, Söderfeldt B and Arnbjerg D: Self - assessment of dental conditions: validity of a questionnaires Community Dent Oral Epidemiol 199I, 19:249-5I.

7. Kallio $P$ and Ainamo J: Self - assessment of gingival bleeding Int Dent J 1990, 40:231-6.

8. Tervonen T, Knuuttila M: Awareness of dental disorders and discrepancy between "objective" and "subjective" dental treatment needs. Community Dent Oral Epidemiol 1988, I 6:345-8.

9. Sgan-Cohen HD, Katz J, Horev T, Dinte A and Eldad A: Trends in caries and associated variables among young Israeli adults over 5 decades Community Dent Oral Epidemiol 2000, 28:234-40.

10. World Health Organization: Oral health surveys - basic methods Geneva: WHO 1987.

II. Joshipura KJ, Pitiphat $W$ and Douglass CW: Validation of selfreported periodontal measures among health professionals Journal of Public Health Dentistry 2002, 62:115-121.

12. Pitiphat W, Garcia RI, Douglass CW and Joshipura KJ: Validation of self-reported oral health measures Journal of Public Health Dentistry 2002, 62:122-8.

13. World Health Organization: Oral health surveys - basic methods Geneva: WHO 1997.
14. Abramson $\mathrm{JH}$ and Abramson $\mathrm{ZH}$ : Survey methods in community medicine Edinburgh: Churchill Livingstone 1999:103.

I5. Shuval JT: Social dimensions of health: The Israeli experience Connecticut: Praeger Publishers 1992: I0I.

16. Schou L and Locker D: Principles of oral health promotion In: Community Oral Health Edited by: Pine MC. Oxford: Wright; 1997: I77186.

17. Daly B, Watt R, Batchelor P and Treasure E: Introduction to the principles of public health In: Essential dental public health Oxford University Press; 2002: I- 17.

\section{Pre-publication history}

The pre-publication history for this paper can be accessed here:

http://www.biomedcentral.com/1472-6831/3/3/prepub
Publish with Bio Med Central and every scientist can read your work free of charge

"BioMed Central will be the most significant development for disseminating the results of biomedical research in our lifetime. "

Sir Paul Nurse, Cancer Research UK

Your research papers will be:

- available free of charge to the entire biomedical community

- peer reviewed and published immediately upon acceptance

- cited in PubMed and archived on PubMed Central

- yours - you keep the copyright
Biomedcentral 